\title{
LA EXPERIENCIA DEL CLIENTE DESDE EL AISLAMIENTO
}

\section{Sciarroni, Roberto}

\section{Resumen:}

En forma repentina se forja un escenario generalizado de anomia que invade a la población y por ende al Consumidor. Fue una etapa de desorganización de la cual no se tenía antecedentes. Las consecuencias fueron inmediatas, sumergió a las personas en una situación caracterizada por la incertidumbre y la confusión. Durante ese fin de semana se migró, de un estado de trabajar y utilizar los excedentes financieros para los placeres del mundo consumista como viajar o divertirse, a una modalidad de trabajar para sobrevivir.

Palabras claves: Repentina - Escenario - Anomia- Consumidor - Incertidumbre Experiencia del Cliente - Customer Experience

\section{Abstract:}

Suddenly a generalized scenario of anomie is forged that invades the population and therefore the Consumer. It was a period of disorganization for which there was no precedent. The consequences were immediate, plunged people into a situation characterized by uncertainty and confusion. During that weekend he migrated from a state of working and using financial surpluses for the pleasures of the consumer world such as traveling or having fun, to a mode of working to survive

Keywords: Suddenly - Scenario - Anomie - Consumer - Uncertainty - Customer Experience

\section{INTRODUCCION}

El 20 de marzo del año 2020, se recordará como un punto de inflexión profundo en la vida de los argentinos. Dos de los periódicos de mayor circulación de la Argentina consignaban títulos hasta ese momento nunca vistos. "Rige la cuarentena obligatoria en todo el país hasta el 31 de marzo"; "El país en cuarentena. Todos deben quedarse en su casa hasta el martes 31. Será delito circular sin justificación". Del día a la noche y en forma repentina se forja un escenario generalizado de anomia que invade a la población y por ende al 
Consumidor. Fue una etapa de desorganización de la cual no se tenía antecedentes. Las consecuencias fueron inmediatas, sumergió a las personas en una situación caracterizada por la incertidumbre y la confusión. Durante ese fin de semana se migró, de un estado de trabajar y utilizar los excedentes financieros para los placeres del mundo consumista como viajar o divertirse, a una modalidad de trabajar para sobrevivir.

\section{LOS CAMBIOS QUE SUCEDIERON}

En efecto cambiaron las regulaciones y los procedimientos habitualmente utilizados perdieron vigencia. Hubo que generar rápidamente nuevas respuestas a problemas inéditos. Se hizo presente, en forma irremediable, una situación de stress insoslayable. Se produjo una sumisión a una carga mayor que la tolerable tanto psíquica como física. Emergieron nuevas reglas de interacción y relacionamiento, y por ende un Consumidor y un modelo de Organización empresarial totalmente distinta.

El trabajo en forma remota, que se multiplicó exponencialmente, y las salidas reducidas solamente a la compra en supermercados, hizo que se produjera un cambio profundo en el comportamiento del Consumidor. En forma repentina los productos se segmentaron naturalmente en dos categorías: los muy necesarios y los completamente prescindibles. El gasto se concentró en alimentos y artículos de limpieza y se minimizó lo que se denomina compra impulsiva. Incrementó el consumo de alcohol para asepsia en el 358\%, el de toallitas desinfectantes en un $179 \%$, los insecticidas en un $175 \%$, la lavandina en un $120 \%$ y los jabones en pan en un $112 \%$. Acompañó este crecimiento las compras de categorías necesarias como las harinas, los polvos para preparar helados y flanes, las pastas secas, los desodorantes de ambientes, el arroz, los productos enlatados y lácteos como el dulce de leche, la manteca y los quesos semiduros y blancos. Emergieron categorías perdedoras como los caramelos, las pastas para limpiar calzados, los aperitivos, las barras de cereales, los budines, las gomas de mascar, los licores y las fragancias entre otras. En marzo del 2020 los Ingresos alcanzaban los $\$ 950.000$ millones de pesos, algo así como $\$ 35.185$ por cada uno de los 27.000 .000 de argentinos en actividad. Rápidamente, el volumen cayó drásticamente en abril, a un monto aproximado de 858.000 millones de pesos, cifra que incluye el Ingreso Familiar de Emergencia. En definitiva, 100.000 millones de pesos menos por mes. El $80 \%$ de los productos que se compraron tuvieron un precio menor a los $\$ 100$. No hay duda que los "cinturones se ajustaron" y los recaudos se profundizaron. 


\section{CONSECUENCIAS SOBRE EL COMPORTAMIENTO.}

Los efectos de lo comentado, poco se hicieron esperar. Podrían sintetizarse en seis secuelas básicas que caracterizan el comportamiento del nuevo Consumidor que nace como producto de la pandemia:

- Mayor nivel de exigencias hacia las marcas:

Se le reclama a las Organizaciones una mayor cercanía al Consumidor. Resurge con notoriedad el principio de Responsabilidad Social Empresaria. La empatía se hace imprescindible, resulta vital estar alineado en los padecimientos del que enfrenta la crisis. El foco se concentra en mantener los precios estables y proveer mayores beneficios. Nace una gran diferencia entre ganadores y perdedores.

- Cambios en la elección de los lugares de compra:

Las compras se circunscriben a los establecimientos cercanos. Se trata de eludir el aglomeramiento de personas. Incrementan en forma exponencial las compras on line. Se asume que a través de ese canal se obtienen mejores precios con un menor riesgo. Se incorporan nuevas categorías al comercio electrónico.

- Cambios en el mix del gasto:

Se prioriza la compra de no perecederos y los productos de limpieza y desinfección.

- Profundización de los miedos:

Los miedos, que aparecen en forma reiterativa, tienden a convertirse en obsesión. Casi el $60 \%$ de una muestra representativa así lo manifiesta. Se percibe, en forma generalizada una fuerte preocupación por la falta de recaudos por parte de la población. Las más preocupadas son las personas de más de 55 años (67\%) y las de mayores niveles socio económicos (60\%). Son altísimos los porcentajes que auguran que la recuperación de la pandemia será lenta y dificultosa.

- Mayor acceso a la información:

Es mucha mayor la exposición a la TV y los diarios. Se consultan, como nunca antes había ocurrido, los sitios WEB de los Organismos Gubernamentales e Internacionales. El streaming emerge como una nueva forma de entretenimiento y crece la exposición a videos y radios online. La infoxicación comienza a dañar las expectativas e incrementar los temores.

- Inmersión en las redes sociales:

Crece en un 69\% la utilización de WhatsApp, en un 52\% el uso de Facebook y en un $51 \%$ el de Instagram. 


\section{FUTURO PERFIL DEL CONSUMIDOR.}

Solamente se podrá gestionar adecuadamente la experiencia del Cliente y, por ende, tratar de satisfacerla si se asumen conjeturas acerca del perfil del mismo, tras el aislamiento.

- Guiado por la ansiedad en las decisiones:

Deberán diseñarse procesos y procedimientos expeditos y amigables. Más que nunca adquiere un especial valor las decisiones rápidas y proactivas.

- Especialmente exigente:

Emergerá un Cliente que es menos susceptible a olvidar los errores. A los productos y a los servicios se les exigirá un proceso de mejora sustancial. Varias de las empresas que operan en el mercado deberán reconstruir la promesa de marca.

- Inteligente en su gasto:

La incorporación de mayor tecnología le posibilitará poseer más habilidad para encontrar con facilidad mayor cantidad de opciones. Tendrá más tiempo para evaluar cada oferta. Será mucho más apto para comparar, justipreciar y elegir entre diferentes alternativas.

- Valorador de la experiencia:

Recompensará a las Organizaciones que lo han hecho bien durante la crisis. Se convertirá en un crítico más profundo del accionar empresario.

- Influenciado por la respuesta social:

Germinará un Consumidor fuertemente influenciado por el comportamiento colectivo.

Serán especialmente tenidas en cuenta las recomendaciones que emanen del imaginario colectivo. La imitación jugará un importante rol.

- Propenso a la automatización:

Las interacciones a distancia jugarán un papel definitorio. Las teleconferencias ya no se alejarán de los usos y costumbres habituales. Se valorarán las reacomodaciones provistas por un autómata que faciliten la vida cotidiana.

- Orientado hacia el aprendizaje en línea:

Crecerá el atractivo por capacitarse y aprender a través de los procesos de elearning. Cada vez se valorizarán más los contenidos con "contenidos". Especialmente los orientados a la práctica cotidiana.

- Inmerso en el teletrabajo:

Contará con una propensión positiva hacia el trabajo en el hogar o a distancia, ya que le otorgará mayores grados de libertad y la posibilidad de auto administrar su labor. 
- $\quad$ Sensible a las experiencias, más que al lujo:

El nuevo Consumidor del pos aislamiento estimará profundamente el vivir experiencias. El sentir la vibración en su piel. Quizá el lujo le dejará espacio al vivir intensamente de lo natural y cotidiano.

- El bienestar y la salud en el centro:

Es muy probable que le otorgue un papel muy importante a la ingesta sana, al conservar un cuerpo saludable y contar con un seguro médico que le trasmita confianza y contención.

- Encaminado hacia un estilo de vida virtual:

Definitivamente, lo que representó internet, el WI Fi y las redes sociales, han integrado hasta a los más escépticos. Muchos de ellos valorizarán profundamente servicios virtuales rápidos, eficientes y amigables. Comprarán cada vez más en el mundo virtual para ahorrar tiempo y cederle espacio a la vida al natural.

- Valorizador del contacto humano:

Intentará recrear y volver a situaciones y experiencias sacrificadas o perdidas. Sera el tiempo de humanizar cada vez más el contacto digital. El desafío será no sólo contactar, sino también conectar.

\section{EL DESAFÍO EMPRESARIAL}

Ante las consecuencias descritas y la aparición de un nuevo tipo de Consumidor, las Organizaciones deberán definir su propio Customer Experience Framework.

En efecto, deberán poner en el Centro de su estrategia al Cliente. Deberán comprender que la realidad está fuera del modelo mental propio. Deberán investigar en forma continua para acceder a conocimientos cada vez más profundos. El Conocimiento del Cliente se convertirá en una ventaja diferencial para la meta estratégica.

Seis son las etapas que se recomienda abordar en forma crítica:

- Conocer al Cliente:

La fórmula será salir de la zona de confort. Intentar nuevas propuestas en forma continua. Crear. Se acabarán los tiempos para los perezosos. Se deberá entender cada vez más al Cliente, se lo deberá sentir, se deberá contar con una actitud proactiva ante los cambios.

- Investigar:

Resultará indispensable precisar los puntos de insatisfacción y las áreas de dolor del Cliente. Definir con precisión como vive sus experiencias. La satisfacción que con 
ellas tiene. A partir de las oportunidades de mejora definir pautas del modelo de Customer Experience a adoptar.

- Conducir:

A partir del modelo definido, se deberá administrar las prácticas y la implementación. Coordinar las actividades. Construir un modelo de follow up que permita tomar las medidas correctivas de forma de alinear dinámicamente las acciones dentro del margen de tolerancia de los objetivos a alcanzar.

- Estrategia:

Se deberá formalizar el modelo de Customer Experience que caracterizará a la Organización. Construir el entramado del ADN de la experiencia que intentará entregar a sus Clientes la Empresa. Edificar la tabla de los mandamientos que orientarán el comportamiento organizacional.

- Diseño:

Resultará categórico escoger dentro de la caja de herramientas, las que se utilizarán para implementar la estrategia definida. Crear para sorprender. Apelar al conjunto de instrumentos que faciliten el desarrollo de su Customer Experience Framework. Entre ellos, mapa de empatía, arquetipos de clientes, medición de la satisfacción de los Clientes, customer journeys, tracking de CX, rastreo de lealtad y segmentación de los clientes, sólo para mencionar algunos.

- Evangelización:

En esencia, resultará imprescindible internalizar el ADN de la organización definido por el modelo. Crear una cultura vigorosa. Formar equipos invencibles. Poner en primer plano la pasión por el Cliente.

La misión no es sencilla pero el desafío es atractivo y los beneficios pueden ser muy importantes. Esta pandemia ha obligado a replantear el valor del conocimiento y la aplicación al trabajo. Habrá que anticiparse o sucumbir en una feroz competencia por luchar palmo a palmo por un nuevo Cliente o retener los existentes.

\section{CONCLUSIONES}

- Se deberá pensar en una nueva propuesta de valor, adecuada a la nueva normalidad. Los mercados finales e industriales manifestaron rápidamente cambios profundos. Cambios, que ineludiblemente, muy lejos de ser coyunturales, serán estructurales y definitivos. 
- El foco deberá centrarse en el proceso de forjar e internalizar un ADN organizacional construido a partir de saber cada vez más del Cliente con el objetivo de ubicarlo en el centro de la estrategia organizacional.

- El vínculo con el Cliente deberá estructurarse a través de la premisa de conectarse, más que comunicarse. Resultará fundamental aprovechar las ventanas estratégicas que abren las amenazas en oportunidades de generar ventajas competitivas sustentables a partir de la lealtad de los clientes.

- Será indispensable una reconversión de la misión y la visión de la empresa, basada en el compromiso de redefinir el negocio, desde el principio de internalizar la pasión por el cliente.

- Seguramente se destacarán los que desarrollen nuevas potencialidades distintivas. Equipos de trabajo empáticos y proactivos y liderazgos participativos serán la clave de los desafíos a desarrollar en la post pandemia.

- La medición en tiempo real las experiencias vividas por los Clientes y la toma de medidas correctivas con rapidez ofrecerá oportunidades estrategias fundamentales a los "Ganadores" en la nueva realidad que se vive.

\section{REFERENCIAS BIBLIOGRÁFICAS:}

Amigo, Adriana. (2017): Negocios con Valor. Una metodología para generar ventajas competitivas sostenibles. EAE. $3^{\circ}$ Edición. $1^{\circ}$ Edición Europea Editorial Académica Española. Madrid. España.

Munuera Alemán, José Luis; Rodríguez Escudero, Ana Isabel. (2007): Estrategias de Marketing. Un enfoque basado en el proceso de dirección. ESIC. Madrid. España.

Carrión Maroto, Juan. (2006): Estrategia. De la Visión a la acción. ESIC. 2 • Edición. Madrid. España.

Ala Pennington, (2016): The Customer Experience Book: How to design, measure and improve customer experience in your business. FT Press. 1ra. Edición 2016. EEUU.

Jeanne Bliss. (2015): Chief Customer Officer 2.0: How to Build Your Customer-Driven Growth Engine. Jossey-Bass 2da. Edición 2015. EEUU. 
Gary Vaynerchuk, (): The Thank You Economy. Harper Business. 1ra Edición 2011. EEUU.

Matthew Dixon, Nick Toman y Rick Delisi. (2013): The Effortless Experience: Conquering the new battleground for customer loyalty. Portfolio. 1ra Edición 2013. Mexico.

Marcos Alvarez (2017): Customer experience. Profit. 1 ra Edición 2017. España.

Karl Wirth, Katie Sweet (2017): One-to-One Personalization in the Age of Machine Learning: Harnessing Data to Power Great Customer Experiences. BookBaby 1ra Edición 2017.

Maurice FitzGerald (2017): Customer-Centric Cost Reduction: How to invest and improve profits without sacrificing your customers (Customer Strategy). SBVV 1ra Edición 2017. EEUU.

Roberto Sciarroni (2019): Desafío en ciernes: poner en primer plano la pasión por el cliente. Revista Mercado. Argentina. 